\title{
REFUERZO DE ESTRUCTURAS CON COMPOSITES AVANZADOS
}

\section{(STRENGTHENING OF STRUCTURESUSING ADVANCED COMPOSITES)}

\author{
Alfonso Recuero, José Pedro Gutiérrez. Instituto Eduardo Torroja-CSIC-Madrid \\ Antonio Miravete, Jesús Cuartero. Dep. Ingeniería Mecánica-Univ. de Zaragoza
}

ESPAÑA

Fecha de recepción: $27-\mathrm{X}-97$

\section{RESUMEN}

La restauración, refuerzo o rehabilitación de estructuras resulta ser uno de los campos de aplicación de mayor interés y más directamente relacionado con los nuevos materiales compuestos. La Industria de la Construcción no ha aceptado aún el uso estructural extenso de los nuevos materiales compuestos, porque todavía no conoce bien cuándo existen ventajas respecto a los materiales tradicionales, tales como el hormigón o el acero. Los profesionales implicados en el proyecto y en la ejecución de obras suelen ser conservadores y resistirse a los cambios. Para aceptar un nuevo material requieren disponer de normativa relativa a la nueva tecnologia, lo que hace necesario que alguna entidad lidere la aceptación del nuevo material o tecnologia. Actualmente no existe ni a nivel nacional ni internacional la experiencia precisa para el desarrollo de tal normativa. Para ello es necesario tener la adecuada base experimental y de realizaciones con éxito.

En el presente trabajo se trata de hacer una introducción al tema y de dar noticia de un proyecto de investigación que se está desarrollando, orientado a proporcionar la base experimental requerida para poner a punto, en un próximo futuro, normas y recomendaciones para el proyecto y la tecnología para el uso de estos nuevos materiales compuestos en el refuerzo de estructuras de edificación y obra civil, atendiendo a su patologia, calidad y durabilidad del refuerzo, y a la estética urbanistica. En él colaboran especialistas en investigación en materiales compuestos y en análisis teórico $y$ experimental y en patologia de estructuras, fabricantes y aplicadores del material y adhesivos, proyectistas y usuarios finales, lo que permitirá considerar todos los aspectos del problema.

PALABRAS CLAVE: materiales compuestos, estructuräs, refuerzo, rehabilitación, análisis experimental, modelos de cálculo, recomendaciones.

\section{SUMMARY}

Restoration, strengthening and rehabilitation of buildings becomes one of the more interesting aspects of the use of composites. Construction industry has not yet accepted the wide structural use of these new materials because it does not know the advantages of composites in comparison with traditional materials, such as concrete or steel. Proffesionals involved in design and construction are conservative and resist to changes. They require codes and specifications, what makes that an entity should lead the use of the new material or technology. At present, the experience needed to prepare those codes does not exist. Experimental tests and successful cases are neccessary for the acceptance of these materials in construction.

In this work, an introduction to the subject is done and notice is given of a project which is developing with the aim to provide the experimental basis, needed to update design codes and standards, and the technology for the use of these new composites in building and civil structures strengthening, taking actual pathology, quality and durability into account, as well as urban aesthetics. Research specialists in composites, structural analysis and testing, and in structural pathology, as well as composites and adhesives manufacturers and appliers, designers and final users will cooperate in this project. This will allow that all relevant aspects of the problem be considered.

KEY WORDS: composites, structures, strengthening, rehabilitation, experimental tests, design models, $\tau$ codes. 


\section{Introducción}

Los materiales compuestos, formados por dos o más materiales de características diferentes que se tratan de conjugar, son usados desde antiguo en la construcción. Como ejemplos típicos pueden citarse el tapial o el hormigón estructural. Los nuevos materiales compuestos empleados en la Construcción, formados por fibras (carbono, aramida, etc.) embebidas en una matriz orgánica (poliéster, epoxi, etc.) se caracterizan por su ligereza (del orden de $1,6 \mathrm{~g} / \mathrm{cm}^{3}$ ), lo cual aporta ventajas tanto desde el punto de vista de economía y facilidad en el transporte de la pieza hasta la obra, como desde el de economía y facilidad en el montaje y puesta en obra, sin olvidar la reducción de cargas muertas cuando estos materiales se utilizan de modo integral o masivo.

Presentan un buen comportamiento ante la corrosión y el ataque de agentes ambientales, lo cual supone una ventaja en aplicaciones costeras, marinas y, en general, en todos aquellos ambientes que sean agresivos, siendo el mantenimiento prácticamente nulo. Otra de sus peculiaridades es la de poseer buenas características mecánicas y, en particular, elevada resistencia mecánica, tanto a tracción como a compresión, cortadura y resistencia al impacto, lo cual permite su utilización en la estructura resistente. Además, no producen interferencias con campos electro-magnéti$\cos$, lo que les hace insustituibles en algunas aplicaciones.

Existen, sin embargo, ciertos inconvenientes que limitan su uso. En muchos de ellos se produce una degradación de sus propiedades a temperaturas no muy elevadas, así como a lo largo del tiempo. Presentan unos coeficientes de dilatación muy diferentes de los del hormigón o el acero. No se conoce suficientemente su interacción con otros materiales, por lo que no existen códigos o recomendaciones de uso de carácter general. Existe una amplia gama de formulaciones, que permite adecuar sus características, pero que dificulta su estandarización o el establecimiento de criterios de control de recepción o de calidad. Su precio unitario es elevado, aunque esto puede compensarse teniendo en cuenta otros aspectos que pueden hacerlos competitivos en el coste total de la obra. Existe, en fin, falta de experiencia en su utilización.

\section{Características mecánicas}

Los materiales del centro de la tabla periódica son poco utilizados como materiales estructurales debido a su carácter quebradizo, una pequeña muesca o microfisura en la superficie puede hacer que provoquen un fallo de forma súbita y prematura en los elementos estructurales de tal material, si bien la importancia de la presencia de un defecto disminuye con el aumento del volumen del material. La presencia de un defecto en una fibrā no tiene por qué suponer un fallo del elemento estructúral, pues cuando la fibra está embebida en una matriz puede colaborar a soportar una carga situada a una corta distancia del defecto.
Entre estos elementos se incluye el carbono, que resulta de interés por sus fuertes enlaces a nivel atómico y resistencia ante agentes químicos y su baja densidad. Las fibras de carbono se consiguen mediante procesos de extrusión de un polímero en un filamento continuo. El filamento es tratado a temperatura de 200 a $300{ }^{\circ} \mathrm{C}$ y luego es sometido a un proceso de carbonización entre 350 y $1.600^{\circ} \mathrm{C}$ en presencia de una atmósfera con gases de $\mathrm{O}, \mathrm{H}$ y N . Las propiedades mecánicas de la fibra resultante pueden ser modificadas a posteriori mediante un proceso térmico donde se alcanzan temperaturas entre 1.300 y $3.000{ }^{\circ} \mathrm{C}$.

Los polímeros reforzados con fibra de carbono parecen los más idóneos para su uso en refuerzo o rehabilitación de estructuras. Para tener un orden de magnitud de sus características, considérese el ejèmplo de una lámina unidireccional con fibras en la dirección axil. Pueden ser descritas básicamente por:

\section{Resistencia}

Módulo elástico

Densidad

Coef. dilatación térmica (long.)

Coef. dilatación térmica (transv.)
$1.482 \mathrm{MPa}$

$115,340 \mathrm{MPa}$

$1,48 \mathrm{~g} / \mathrm{cm}^{3}$

$0,23 \cdot 10^{-6} \mathrm{~m} /{ }^{\circ} \mathrm{C}$

$34,0 \cdot 10^{-6} \mathrm{~m} /{ }^{\circ} \mathrm{c}$
Comercialmente alcanzan un módulo elástico de $230 \mathrm{GPa}$, pudiendo llegar en elementos especiales a módulos altos que alcanzan valores de 480 a $700 \mathrm{GPa}$, mientras que con fibras perfectamente alineadas pueden alcanzar los $1.000 \mathrm{GPa}$.

No deben dejarse de lado otros tipos de fibras, que, aunque puedan ser más interesantes para obra nueva por ser más económicas que las de carbono, también pueden usarse en refuerzo de estructuras.

En la tabla 1 pueden verse características mecánicas de algunasfibras de interés para materiales compuestos de uso estructural. El gran incremento en importancia de estas fibras compuestas se debe a que las propiedades individuales de los materiales pueden ser combinadas, obteniendo propiedades que se conseguirían con gran dificultad y con un alto coste para materiales convencionales. Estos materiales proporcionan la posibilidad de entremezclar fibras y sistemas de matrices e incluir otros tipos de fibras (fibras híbridas), creando un amplio campo de aplicación.

En los últimos años se ha experimentado un auge importante en el desarrollo de nuevas tecnologias textiles aplicables a materiales compuestos. Por medio de estas nuevas tecnologías textiles, además de conservarse las ventajas derivadas de un buen diseño, aprovechando la anisotropía de las propiedades en el plano de los materiales compuestos tradicionales, se mejoran sustancialmente las propiedades fuera del plano, débiles en los apilamientos tradicionales. Dentro de estas tecnologías se encuentran el trenzado o braiding, tejido entramado o weft knitting, tejido en urdimbre o warp knitting y el cosido multicapa. 
TABLA 1

Características mecánicas de algunas fibras

\begin{tabular}{|c|c|}
\hline \multicolumn{2}{|c|}{ VIDRIO E } \\
\hline DIÁMETRO DEL HILO (mm) & $10-20$ \\
\hline TENSIÓN ROTURA (GPa) & $3,6 / 2,6-3,4$ \\
\hline MÓDULO TRACCIÓN (GPa) & 76 \\
\hline TENSIÓN ROTURA ESPECÍFICA (GPa) & $1,40 / 1,34$ \\
\hline MÓDULO ESPECÍFICO & 29 \\
\hline DENSIDAD $\left(\mathrm{g} / \mathrm{cm}^{3}\right)$ & $2,56 / 2,55$ \\
\hline COEFICIENTE DE EXPANSIÓN TÉRMICA $\left(10^{6}{ }^{\circ} \mathrm{C}\right)$ & 4,9 \\
\hline CALOR ESPECÍFICO kJ/kg $\mathrm{K}$ & 0,7 \\
\hline \multicolumn{2}{|c|}{ KEVLAR 29} \\
\hline TENSIÓN ROTURA (GPa) & 2,76 \\
\hline MÓDULO TRACCIÓN (GPa) & 58 \\
\hline TENSIÓN ROTURA ESPECÍFICA (GPa) & 1,92 \\
\hline MÓDULO ESPECÍFICO & 40 \\
\hline DENSIDAD $\left(g, 2 \mathrm{~m}^{3}\right)$ & 1,44 \\
\hline \multicolumn{2}{|c|}{ KEVLAR 49} \\
\hline TENSIÓN ROTURA (GPa) & $2,94 / 3-3,6$ \\
\hline MÓDULO TRACCIÓN (GPa) & 128 \\
\hline TENSIÓN ROTURA ESPECÍFICA (GPa) & $2,48 / 2,03$ \\
\hline MÓDULO ESPECÍFICO & 90 \\
\hline DENSIDAD $\left(\mathrm{g} / \mathrm{cm}^{3}\right)$ & 1,45 \\
\hline DIÁMETRO DEL HILO (mm) & 12 \\
\hline
\end{tabular}

WEFT KNITTING es la técnica más usada en la industria de confección textil. Los tejidos se realizan a partir de una única hebra que va formando sucesivas curvas con los movimientos de las agujas. Las diferentes agujas pueden controlarse de manera independiente, consiguiéndose multitud de variaciones en la arquitectura del tejido, que se va formando transversalmente fila a fila. La disposičión de las fibras en múltiples curvas confiere al tejido uña elevada capacidad de deformación, permitiendo la elaboración de preformas complejas con dobles curvaturas que pueden

(c) Consejo Superior de Investigaciones Científicas adaptarse perfectamente al molde, para, finalmente, ser convertidas en el componente final, tras un proceso de inyección de resina.

Esta disposición del tejido en forma de curvas reduce las propiedades mecánicas finales del tejido, reducción que obedece a motivos geométricos y al daño que se produce en las fibras al curvarlas, daño que es especialmente importante en las fibras de carbono. Así, las propiedades en el plano obtenidas en materiales compuestos reforzados con 
estos tejidos, son inferiores a las obtenidas con laminados convencionales con los mismos porcentajes de fibra, sin embargo, su deformación de rotura y, por tanto, su resistencia a impacto, es mucho mayor. Los desarrollos actuales de estas tejedurias se centran en la incorporación al tejido base de fibras rectas en distintas direcciones para mejorar las propiedades mecánicas.

E1 COSIDO MULTICAPA es una técnica que posibilita la unión de múltiples láminas de refuerzo (unidireccionales o tejidos), mejorándose substancialmente las propiedades fuera del plano, especialmente la resistencia a cortante interlaminar.

La forma en que estos materiales llegan a la obra puede ser:

- en fibras o telas preimpregnadas en monómero, para ser aplicadas y curadas in situ.

- en bandas longitudinales para ser pegadas al elemento a reforzar.

- en barras y cables para armar o pretensar.

- en perfiles pultrusionados.

- en elementos especiales, con zonas de fibras o zonas de telas, reforzados en las zonas adecuadas y curados en fábrica.

- en rejillas formadas por "costillas" de poliéster y fibra de vidrio.

- en grandes piezas moldeadas.

Las caracteristicas de estos materiales dependen, principalmente, del tipo, riqueza y orientación de las fibras, pero también de la matriz y del proceso de curado. Para situar el orden de magnitud, en la tabla 2 pueden verse algunos datos comparativos de las características de materiales utilizados en construcción. En la tabla 3 pueden verse datos de distintos tipos de laminados con fibra de vidrio para usos estructurales y, en la tabla 4 , datos de fibras de carbono preimpregnadas.

En las figuras 1 y 2 se muestran los diagramas tensióndeformación del hormigón, del acero y de CFRP. La figu-

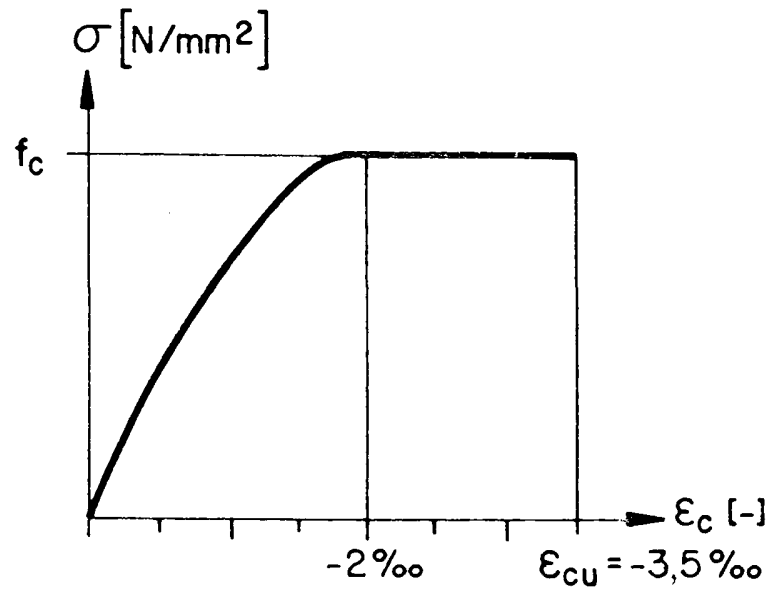

Fig. 1.- Diagrama tensión-deformación del hormigón.

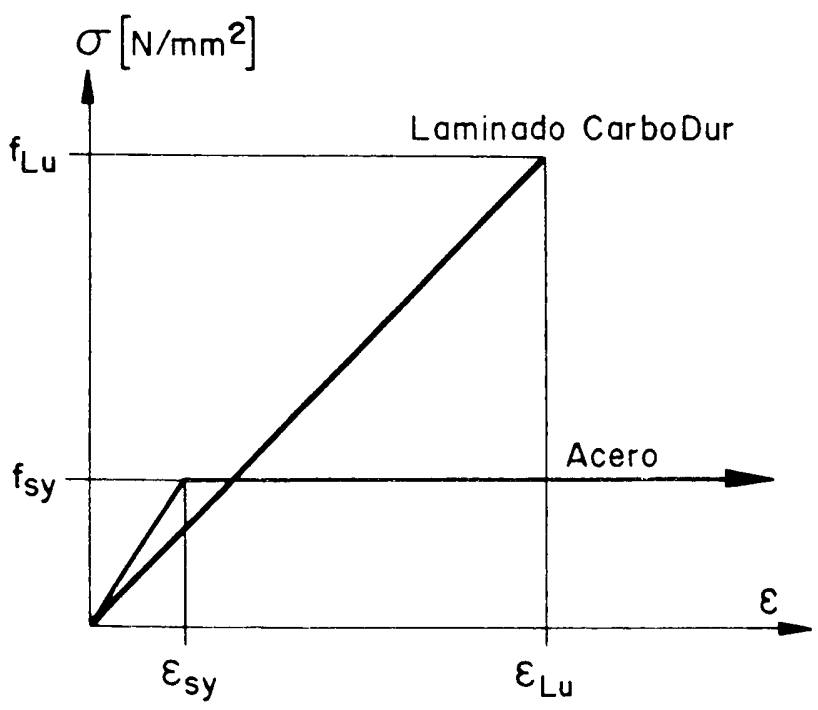

Fig. 2.- Diagramas tensión-deformación del acero y de un laminado CarboDur.

ra 3 recoge los diagramas tensión-deformación para varios tipos de laminados. La característica más notable de estos últimos es su permanencia en régimen elástico hasta

TABLA 2

Propiedades de varios materiales utilizados en tuberías y depósitos

\begin{tabular}{||l|c|c|c|c||}
\hline \multicolumn{4}{||c|}{ PROPIEDADES DE VARIOS MATERIALES UTILIZADOS EN TUBERIAS Y DEPÓSITOS } \\
\hline Tipo de Material & $\begin{array}{c}\text { Densidad D } \\
\left(\mathbf{g} / \mathbf{c m}^{3}\right)\end{array}$ & $\begin{array}{c}\text { Coeficiente de } \\
\text { Dilatación }\end{array}$ & $\begin{array}{c}\text { Tracción } \\
\text { (MPa) }\end{array}$ & $\begin{array}{c}\text { Compresión } \\
\text { (MPa) }\end{array}$ \\
\hline Hormigón & $2,1-2,2$ & $12 \cdot 10^{-6}$ & $\cdots$ & $20-40$ \\
\hline Hierro & 7,85 & $12 \cdot 10^{-6}$ & $220-400$ & $220-400$ \\
\hline Resina poliéster & $1,2-1,4$ & $80-100 \cdot 10^{-6}$ & $45-60-$ & $150-200$ \\
\hline Fibra vidrio & 2,55 & $4,6 \cdot 10^{-6}$ & $3.500^{-}$ & $\cdots$ \\
\hline Composite & $1,5-2,1$ & $1,25 \cdot 10^{-6}$ & $120-600$ & $\ldots$ \\
\hline \hline
\end{tabular}


TABLA 3

Características de laminado de fibra de vidrio y resina EPOXI-MAT

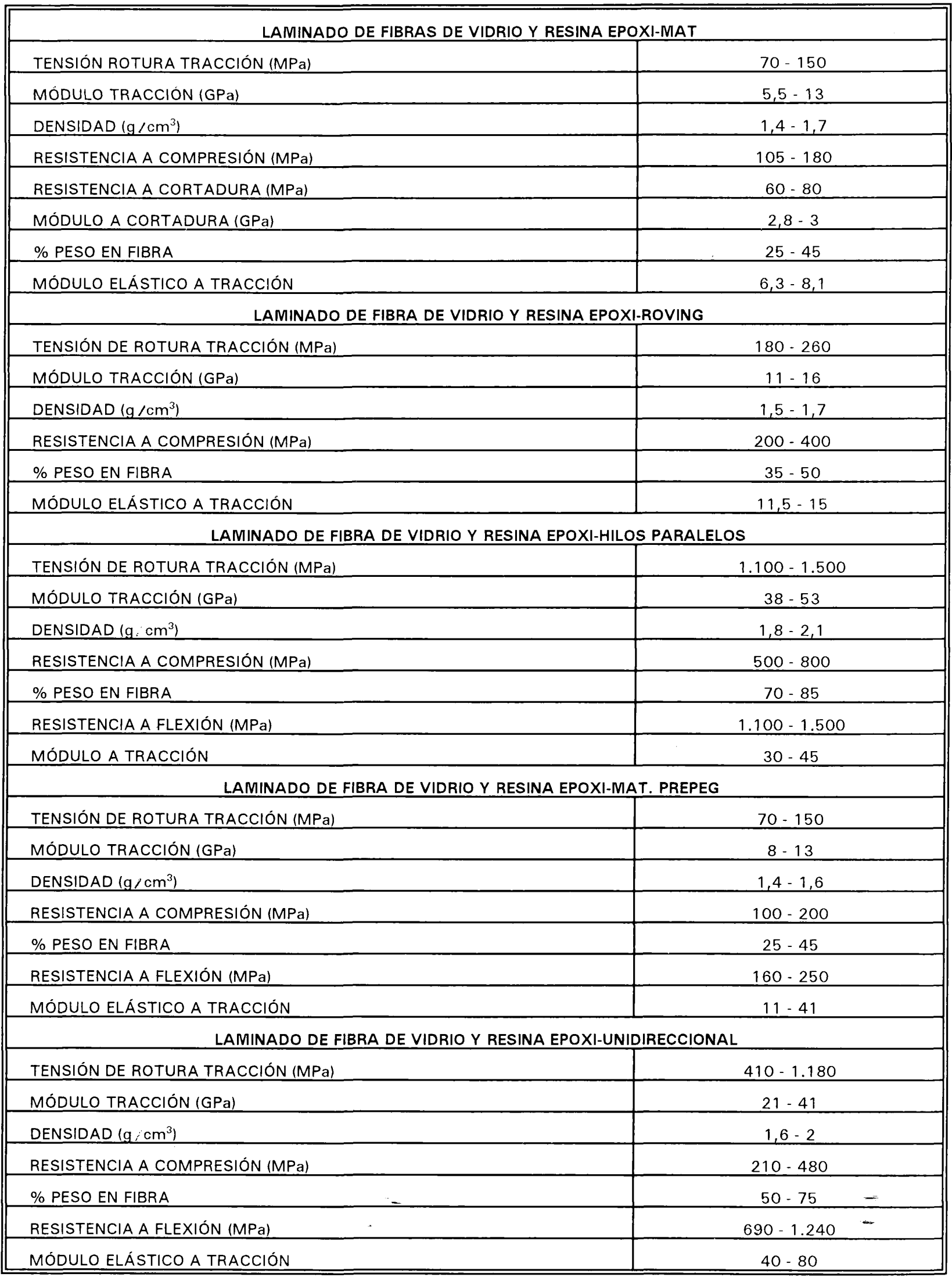


TABLA 4

Datos de láminas de fibra de carbono preimpregnadas, fabricadas por Mitsubishi

\begin{tabular}{||c|c|c|c||}
\hline \multicolumn{2}{|c|}{ DATOS PARA USO DE LÁMINAS DE FIBRA DE CARBONO PREIMPREGNADAS } \\
\hline $\begin{array}{c}\text { TIPO DE } \\
\text { MATERIAL }\end{array}$ & $\begin{array}{c}\text { ESPESORES } \\
(\mathrm{mm})\end{array}$ & $\begin{array}{c}\text { RESISTENCIA A } \\
\text { TRACCIÓN (MPa) }\end{array}$ & $\begin{array}{c}\text { MÓDULO DE YOUNG } \\
\text { (MPa) }\end{array}$ \\
\hline REGULAR RM & $0,097 \mathrm{a} 0,167$ & 2.500 & 240.000 \\
\hline ALTO MÓDULO HM & 0,095 & 2.000 & 650.000 \\
\hline
\end{tabular}

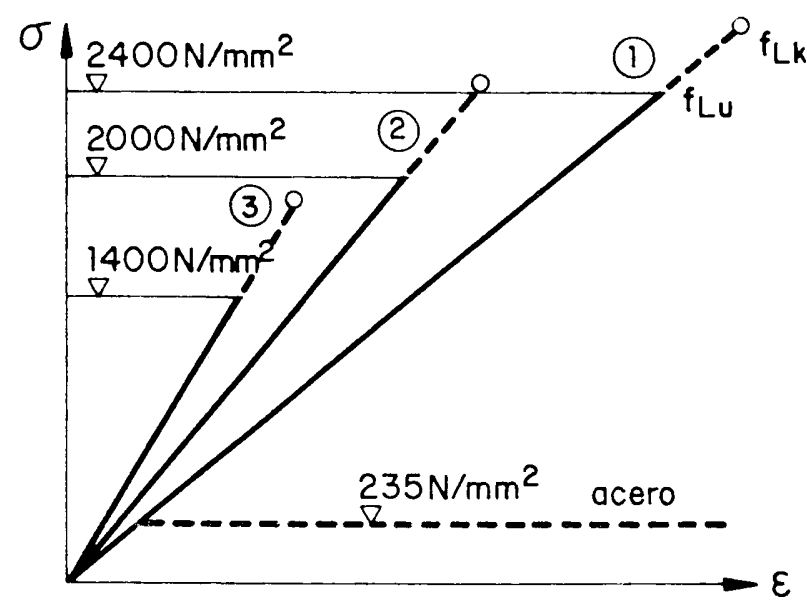

Fig. 3.- Diagramas tensión-deformación de distintos tipos de laminados Sika CarboDur. rotura, sin presentar plastificación. Esto hace que su rotura sea frágil. Se están realizando estudios sobre la utilización de fibras cortas para tratar de conseguir materiales con un comportamiento elasto-plástico.

Entre las presentaciones comerciales disponibles en España, pueden citarse las bandas de CFRP fabricadas por Sika. Se presentan en rollos de $50 \mathrm{~mm}$ de ancho y $1,2 \mathrm{~mm}$ de espesor. Las fibras están alineadas paralelamente de forma longitudinal mediante un proceso de pultrusión y adheridas por resina epoxi; los valores de los esfuerzos de tracción son pequeños, porque existe un gran número de fibras unidireccionales. Se usan diferentes fibras de carbono para permitir la obtención de tiras de CFRP con diferentes módulos de elasticidad. En la tabla 5 se recogen datos de estas bandas.

TABLA 5

Características de bandas de CFRP por Sika

\begin{tabular}{|c|c|c|c|c|}
\hline \multicolumn{5}{|c|}{ TABLA COMPARATIVA DE LOS DISTINTOS MATERIALES } \\
\hline $\begin{array}{c}\text { TIPO DE } \\
\text { MATERIAL }\end{array}$ & $\begin{array}{c}\text { MÓDULO DE } \\
\text { YOUNG } \\
\left\langle\mathrm{N} / \mathrm{mm}^{2}\right\rangle\end{array}$ & $\begin{array}{c}\text { RESISTENCIA A } \\
\text { TRACCIÓN } \\
\left(\mathrm{N} / \mathrm{mm}^{2}\right)\end{array}$ & $\begin{array}{c}\text { TENSION DE } \\
\text { ROTURA } \\
\left.\text { (N/mm }{ }^{2}\right)\end{array}$ & $\begin{array}{c}\text { ELONGACIÓN DE } \\
\text { ROTURA } \\
(\%)\end{array}$ \\
\hline $\begin{array}{l}\text { SIKA } \\
\text { CARBODUR S }\end{array}$ & 155.000 & $>2.400$ & 3.100 & $>1,9$ \\
\hline $\begin{array}{l}\text { SIKA } \\
\text { CARBODUR M }\end{array}$ & 210.000 & $>2.000$ & 2.400 & $>1,1$ \\
\hline $\begin{array}{l}\text { SIKA } \\
\text { CARBODUR H }\end{array}$ & 300.000 & $>1.400$ & 1.600 & $>0,8$ \\
\hline
\end{tabular}

TABLA 6

Comparación entre las propiedades de cables parafil con acero para pretensado

\begin{tabular}{|c|c|c|c|}
\hline TIPO DE MATERIAL & $\begin{array}{c}\text { RESISTENCIA A } \\
\text { TRACCIÓN (GPa) }\end{array}$ & $\begin{array}{c}\text { MÓDULO DE } \\
\text { ELASTICIDAD (GPa) }\end{array}$ & 12 \\
\hline CABLE PARAFIL TIPO A & 0,62 & 77,5 \\
\hline CABLE PARAFIL TIPO F & 1,93 & 126,5 \\
\hline CABLE PARAFIL TIPO G & 1,93 & 0,53 \\
\hline ACERO PRETENSADO & 1,49 & 200 \\
\hline
\end{tabular}




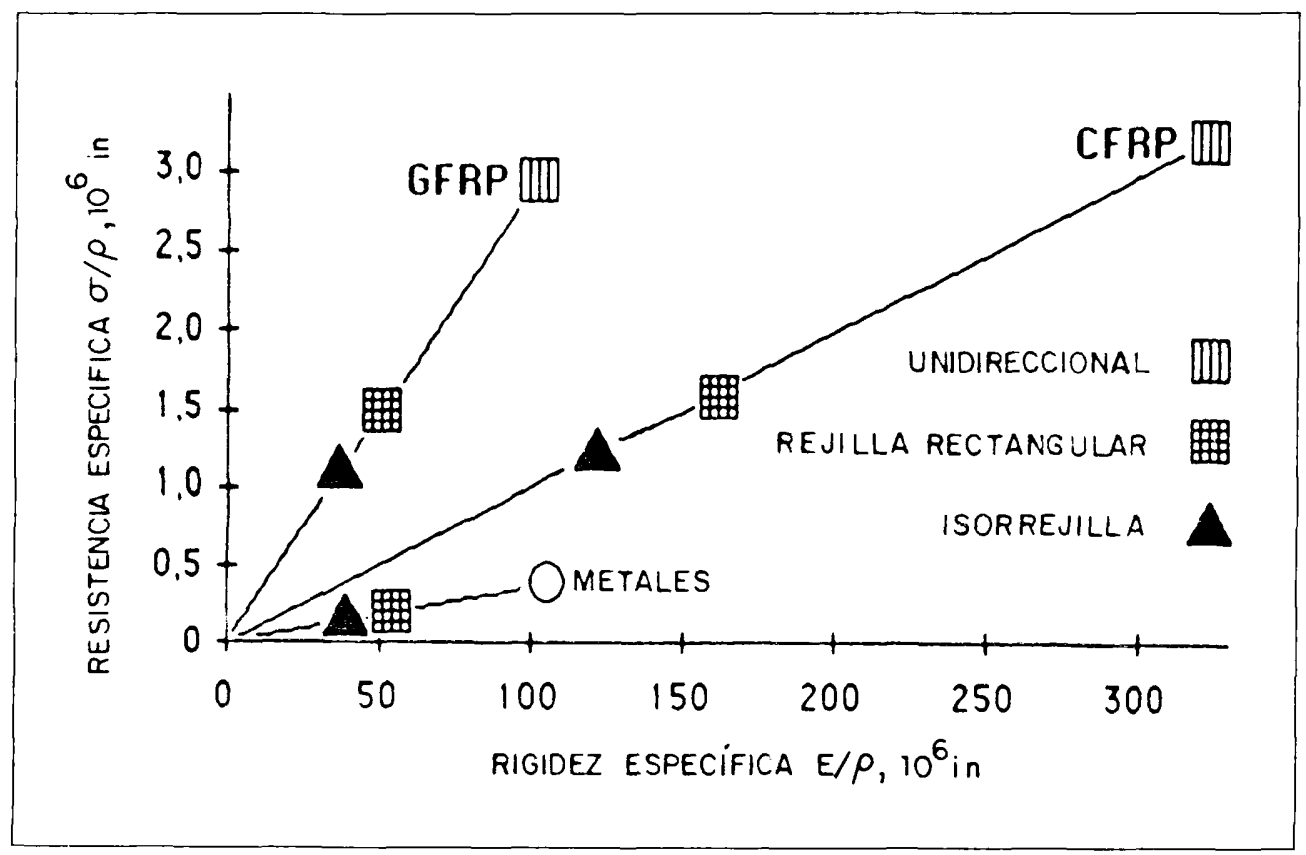

Las propiedades unidireccionales de las rejillas de GFRP aventajan a las propiedades isotrópicas del metal.

Fig. 4.- Rejillas metálicas VS rejillas de GFRP.

Respecto a cables, el sistema EMPA consta de unos hilos de T300 fibras y matriz de epoxi y para grandes estructuras T700 fibras y un sistema también de resina epoxi. Los cables de CFRP están constituidos por la unión de los mencionados hilos. Los diseños considerados para cables hechos de hilos de CFRP unidireccionales, son similares a la de los cables de acero con una pequeña excepción debida a la alta anisotropia natural del material. Se intenta que los hilos, embebidos en una matriz polimérica, estén protegidos ante el impacto, evitando también las posibles fricciones entre los propios hilos y buscando protección ante las radiaciones UV. En la tabla 6 pueden verse características de cables y su comparación con los de acero.

En la figura 4 se muestran diagramas comparativos de valores específicos (por unidad de masa) de distintos materiales empleados en construcción, incluyendo distintas configuraciones de enrejillados de poliéster reforzado con fibra de vidrio, según los ángulos que formen las costillas del enrejillado.

\section{Refuerzo de estructuras}

Debe resaltarse la importancia -cada vez mayor- que tiene el mantenimiento del parque urbanístico, tanto histórico como reciente. En muchos casos, el coste es secundario frente a la calidad, estética y durabilidad del resultado. En todas las ocasiones es importante reducir las molestias inherentes a la realización de obras en edificios en funcionamiento. La restauración, refuerzo o rehabilitación de estructuras resulta ser uno de los aspectos de mayor interés y más directamente relacionado con los nuevos materiales compuestos. La resistencia, ligereza y el comportamiento antc los agentes climáticos y la corrosión hacen de estos materiales unos serios candidatos para su utilización en este tipo de proyectos. Canadá, Japón y EE.UU son los países en los que más se ha avanzado en este campo.

En Europa, Suiza, Alemania e Italia han hechonumerosas realizaciones de refuerzo de estructuras, principalmente puentes y edificios históricos. 
Entre las varias formas de aplicación de estos nuevos materiales compuestos en el refuerzo o rehabilitación de estructuras pueden citarse:

- Sustitución del armado corroído y el hormigón que le rodea. Las reparaciones consisten en sustituir las armaduras corroídas y el hormigón que lo rodea por una nueva capa de hormigón a la que se une una lámina de materiales compuestos de matriz termoestable.

- Suplemento a la armadura existente, con objeto de mejorar la capacidad a flexión, cortante, o la ductilidad del elemento reforzado. Pueden utilizarse láminas de poliéster reforzado con fibra de vidrio/poliéster o de epoxi reforzada con fibras de carbono. Estas últimas, más rígidas y resistentes que las de fibra de vidrio, aunque son más caras que el acero, son más manejables, por lo que el coste de su puesta en obra es menor.

- Colocación de perfiles de fibra de vidrio. Los perfiles pultrusionados están especialmente indicados para restauración, ya que al estar todas las fibras en la dirección del perfil, la resistencia y rigidez son muy elevadas.

- Pretensado exterior de la estructura. Pueden utilizarse cables o barras de material compuesto, o elementos especialmente diseñados preparados para su pretensado con sistemas habituales.

Utilización de enrejillados formando superficies tridimensionales constituidas por "costillas" lineales de poliéster reforzado con fibra de vidrio.

Un tipo de refuerzo habitual es el de flexión pegando bandas preimpregnadas o rígidas en las zonas de tracción. E1 5\% de los puentes deteriorados en Europa pueden ser reforzados de esta forma empleando los nuevos materiales compuestos, en lugar de las convencionales láminas de acero. En España ya se han realizado algunos refuerzos de este tipo. Estos materiales en un ambiente agresivo muestran una buena conducta a lo largo del tiempo, mientras que el acero acaba por corroerse. Otra desventaja que presentan las láminas de acero es su limitada longitud (raramente se sobrepasan los 6-8 m), mientras que con estos materiales se puede conseguir grandes longitudes.

Otro tipo de refuerzo utilizado es el zunchado de pilas o pilares, envolviéndoles en bandas de fibras preimpregnadas. En 1981 se modificó la normativa USA respecto al proyecto antisísmico de los puentes, multiplicando por 8 la armadura transversal de las pilas. Por este motivo, se ha utilizado este tipo de refuerzo en parte de los puentes de California, con objeto de mejorar la ductilidad de las pilas ante acciones sísmicas. Una vez curado, el material se pintaba por estética y para proteger la matriz de la acción de los rayos UV.
Un campo claro de utilización es el de estructuras en ambientes marinos o sumergidas. La armada USA ha utilizado este tipo de refuerzo en muelles de atraque, debido al uso de grúas móviles de gran capacidad y al aumento del tamaño de los barcos. Están realizando estudios experimentales al respecto en sus instalaciones de San Diego.

También se han utilizado bandas rigidas de CFRP en el refuerzo de estructuras antigüas de ladrillo sometidas a distintos tipos de esfuerzo.

Otro campo de interés es el de cables u otros elementos pretensados. De hecho, se está estudiando la forma de pretensar bandas rígidas de CFRP, con objeto de mejorar el aprovechamiento de su capacidad resistente. Los cables para pretensar se vienen usando hace algunos años. Su comportamiento a corto plazo es satisfactorio, aunque el comportamiento a largo plazo está aún en experimentación. Éstos se usan principalmente en Japón y en Europa, aunque sólo pequeñas compañías ofrecen cables apropiados, donde los sistemas de anclaje pueden cumplir con los requerimientos estáticos. Se pueden identificar dostipos de anclajes, el primero en los que la carga es introducida por fuerzas entre la matriz y el tendón compuesto y el segundo tipo es similar al de tendones de acero convencional, donde la fuerza es introducida por fricción. Hay algún otro sistema que combina el caso de anclajes mecánicos y adhesivos.

\section{Unión del refuerzo a la estructura}

La unión del refuerzo de FRP a la estructura, al igual que con otros tipos de refuerzo, puede hacerse mediante pegado, que es el caso más habitual, o mediante conexiones mecánicas. Estas últimas pueden darse en elementos especiales en los que se refuerza adecuadamente las zonas en que se va a realizar la conexión mecánica (tornillos, pasadores, anclajes, etc.) para que resistan las tensiones producidas.

En la tabla 7 pueden compararse las ventajas y desventajas de las uniones adhesivas frente a las uniones mecánicas

En el caso de fibras o telas preimpregnadas, el propio monómero de impregnación actuará como pegamento, en tanto que con los elementos fabricados el método habitual de unión con la estructura existente es mediante pegado. En consecuencia, uno de los aspectos importantes en el uso de estos materiales compuestos es la selección del adhesivo adecuado. Dado que los materiales para la construcción son de naturaleza muy variaday abarcan hormigón, piedra, metales, madera, plásticos, vidrio, etc., existe una diversidad de adhesivos orientados a unirlos, técnica y económicamente, del modo más apropiado. 
TABLA 7

Sistemas estructurales

\begin{tabular}{|c|c|c|}
\hline SISTEMA & VENTAJAS & DESVENTAJAS \\
\hline EPOXI & $\begin{array}{l}\text { Alta adhesión final. } \\
\text { Durabilidad. } \\
\text { Cualquier espesor. } \\
\text { Contracción del } 2 \% \text {. }\end{array}$ & $\begin{array}{l}\text { Preparación de superficies } \\
\text { Vida de la mezcla. } \\
\text { Ciclos de curado. } \\
\text { Dosificación y mezcla de los componentes. }\end{array}$ \\
\hline POLIETURANOS & $\begin{array}{l}\text { Flexibilidad. } \\
\text { Mejor para plásticos. }\end{array}$ & $\begin{array}{l}\text { Menor adhesión final que los epoxis. } \\
\text { Peor para cristal y metales. }\end{array}$ \\
\hline ACRÍLICOS & $\begin{array}{l}\text { Buena adhesión final. } \\
\text { Fácil aplicación. } \\
\text { Curado rápido. } \\
\text { Preparación de } \\
\text { superficies. }\end{array}$ & $\begin{array}{l}\text { Espesor de la capa. } \\
\text { Contracción del } 7 \% . \\
\text { Durabilidad. }\end{array}$ \\
\hline
\end{tabular}

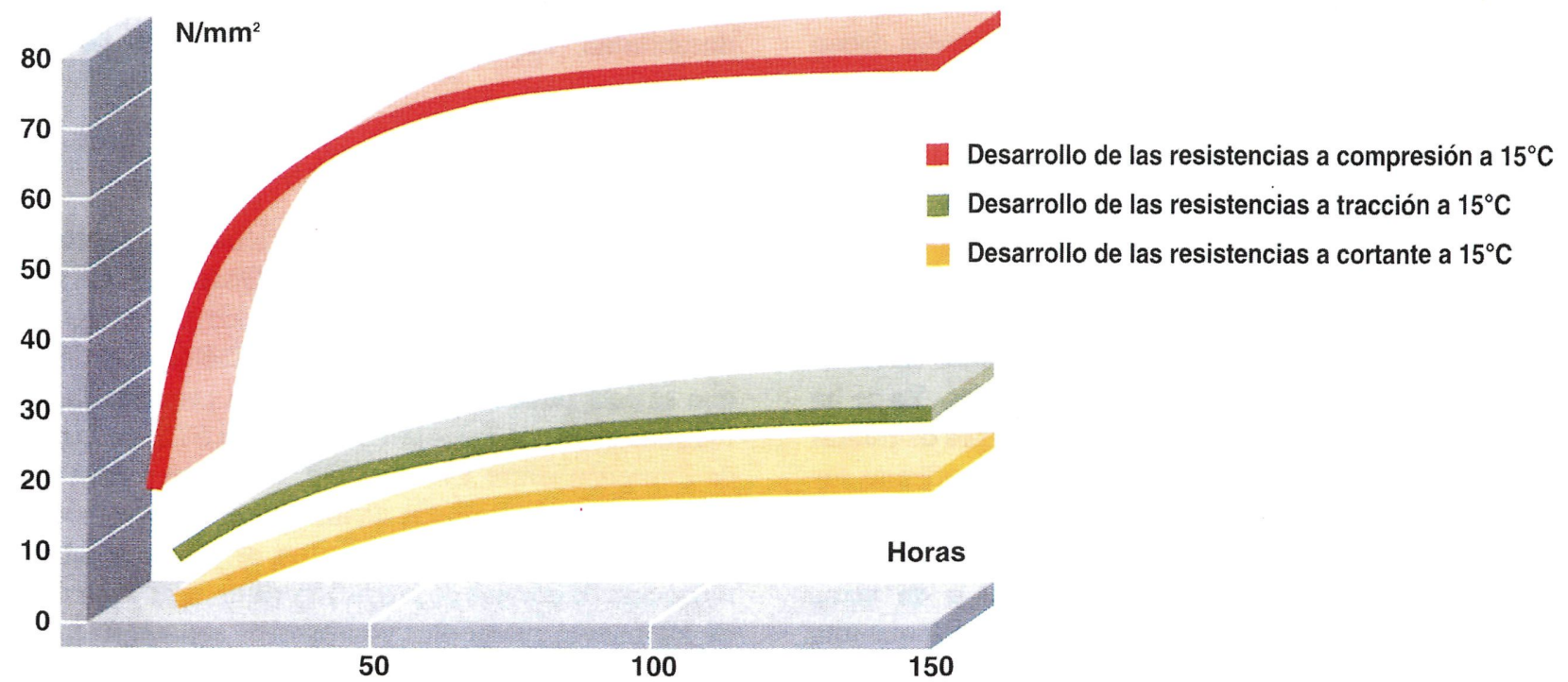

Fig. 5.- Desarrollo de las resistencias del adhesivo Sikadur ${ }^{\circledR}-30$

Se entiende como adhesivo estructural aquél que, usado en combinación con un material estructural, trabaja de modo que la junta o línea de encolado no falle cuando el material trabaje a tensiones de hasta su límite elástico y permita que la estructura trabaje según sus requerimientos mecánicos y físicos. Atendiendo a un criterio resistente puramente numérico, resulta difícil encontrar la fronteraentre adhesivo estructural y no estructural, pudiendo definirse como estructural aquél que supere, a temperatura ambiente, los $10 \mathrm{MPa}$ de resistencia a tracción. En la figura 5 se muestra una curva de evolución de la resistencia de un adhesivo con el tiempo.

\section{Cálculo del refuerzo}

Como puede verse en la figura 2, los FRP presentan un comportamiento elástico y lineal hasta rotura, lo que les

(c) Consejo Superior de Investigaciones Científicas Licencia Creative Commons 3.0 España (by-nc) confiere un comportamiento frágil. Esta falta de aviso previo a la rotura se refleja en los coeficientes de seguridad a adoptar y en los requerimientos de capacidad residual de la estructura a reforzar. Por otra parte, la conexión de elementos de estos materiales entre sí o con la estructura anterior suele ser semi-rígida, lo que complica su análisis, llegándose a considerar el uso de tablas. Las reglas de cálculo dependerán del material (de su rigidez, deformabilidad, de la forma de unión, de las características del ambiente en que va a estar, etc.). En general, los fabricantes de los productos proponen normas de cálculo. Debe señalarse que los borradores de normas para estructuras de materiales compuestos de Japón y de Canadá no incluyen el refuerzo de estructuras, sino sólo estructuras originales.

Por vía de ejemplo, veamos algunas indicaciones para el 
cálculo de refuerzos con bandas de CFRP. En el caso de refuerzo a flexión, la banda puede considerarse como elemento que puede trabajar a tracción. Para estudiar su comportamiento se puede utilizar la analogía de la celosía. En consecuencia, en el caso de vigas o placas serán necesarios estribos externos anclados en la zona de compresión, que, en algunos casos, podrán sustituirse por platabandas de acero pegadas.

Puesto que las láminas de CFRP no llegarán habitualmente a los extremos, tendrán que ser ancladas en zonas de tracción. La longitud de anclaje, dependiente de los materiales de refuerzo y a reforzar, del adhesivo y del tipo de solicitación, se calcula mediante fórmulas empíricas.

Se ha podido comprobar experimentalmente que el cálculo a flexión de vigas o placas reforzadas con bandas puede hacerse siguiendo las reglas aplicables al hormigón armado. Así, se supone que la deformación de la sección plana es también plana, pese a que no es cierto para la capa de adhesivo. Las deformaciones límite de las bandas deben elegirse de modo que se evite la separación prematura de la banda del hormigón. También son aplicables las reglas de cálculo del hormigón respecto al cortante. Adicionalmente, hay que comprobar otras posibles roturas de la banda, del adhesivo, despegue o la delaminación de las bandas.

Otro aspecto a contemplar es la influencia de las variaciones térmicas y de las temperaturas alcanzadas. Ya se ha mencionado la diferencia entre los coeficientes de dilatación térmica entre el hormigón y los FRP, y además los polímeros pueden cambiar sus características, a veces de forma irreversible, al pasar de ciertas temperaturas. Esto puede llevar a la necesidad de pinturas o de otros recubrimientos en casos de intemperie. Piénsese que una banda con fibra de carbono es casi un cuerpo negro y que expuesta al sol puede adquirir temperaturas muy elevadas. Estas consideraciones se refieren tanto al FRP como al adhesivo.

\section{Aceptación de estos materiales}

La Industria de la Construcción no ha aceptado aún el uso estructural extenso de los nuevos materiales compuestos, porque todavía no conoce bien sus ventajas respecto a los materiales tradicionales, tales como el hormigón oel acero. Los profesionales implicados en el proyecto y en la ejecución de obras suelen ser conservadores y resistirse a los cambios. Para que un nuevo material sea aceptado es necesario disponer de normativa relativa a la nueva tecnología, lo que hace necesario que alguna entidad lidere la aceptación del nuevo material o tecnología. Actualmente no existe la experiencia precisa para el desarrollo de tal normativa. Muestra del interés por el tema es la reciente creación de un grupo de trabajo sobre el mismo, dentro de la asociación GEHO-ATEP. La Asociación Española de

(c) Consejo Superior de Investigaciones Científicas Licencia Creative Commons 3.0 España (by-nc)
Materiales Compuestos (AEMAC), a la que pertenecen tanto el Instituto Eduardo Torroja como el Departamento de Ingeniería Mecánica de la Universidad de Zaragoza, se ocupa de los avances en la utilización y el desarrollo de estos materiales, principalmente en Ingeniería Mecánica, como lo muestran los proceedings de su último congreso, celebrado en Madrid en diciembre de 1997, en los que sólo aparece una contribución relativa a su uso en construcción.

Para que se produzca la aceptación de estos materiales estructurales en construcción es necesario tener la adecuada base experimental y de realizaciones con éxito. Entre las aplicaciones más susceptibles de tener éxito están aquéllas en las que se haga uso de la resistencia a la corrosión y de su mayor facilidad de aplicación, que superen la actual desventaja de su alto precioy de la falta de especificaciones. Es necesario conocer los mecanismos de transferencia de cargas de los elementos estructurales antiguos a los nuevos, su evolución con el tiempo y las condiciones ambientales, forma correcta de proyectar y de aplicar el refuerzo, durabilidad, implicaciones económicas y de puesta en obra.

\section{Proyecto en curso}

La CICYT ha aprobado la financiación del proyecto MAT 96/1218, titulado "MATERIALES COMPUESTOS PARA SU USO EN REFUERZO O REHABILITACION DE ESTRUCTURAS DE EDIFICIOS Y DE OBRA CIVIL", que se está desarrollando, de manera coordinada, en el Instituto Eduardo Torroja-CSIC y en la ETSI Industriales de Zaragoza, entre 1997 y 1999. El objetivo general del proyecto es la adquisición de los conocimientos teóricos y experimentales que permitirán en el próximo futuro la redacción de normas de proyecto y de la tecnología del uso de los nuevos materiales compuestos contemplada en el proyecto en la reparación y refuerzo de estructuras (Foto 1). Los objetivos parciales son:

- Analizar experimentalmente las propiedades mecánicas y su evolución con el tiempo y en distintas condiciones

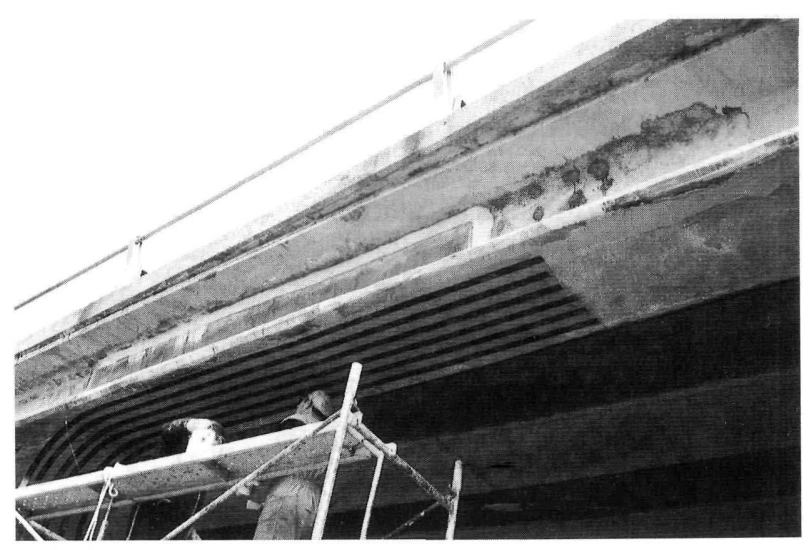

Foto 1.- Reparación de una viga de puente. 
ambientales, de distintos materiales compuestos existentes y de nuevo diseño para su utilización en el refuerzo o reparación de estructuras.

- Estudiar las técnicas de aplicación de refuerzos de acuerdo con la patología, el tipo de elemento estructural o de estructura, su forma de colaborar con la estructura existente y mecanismos de fallo.

- Estudiar la aplicabilidad de las técnicas de pretensado a barras y cables de materiales compuestos o proponer la adaptación de las mismas.

- Analizar experimentalmente el uso de los adhesivos estructurales, determinando los parámetros más adecuados para su uso en función de las solicitaciones actuantes.

- Proponer modelos de reglas de buena práctica en la elección y en la correcta aplicación de los materiales de refuerzo y de los adhesivos estudiados en el proyecto.

- Proponer modelos de cálculo de estructuras que incluyan la colaboración entre ambos materiales y validarlos experimentalmente.

Para despertar el mayor interés posible, está previsto aplicar estos materiales en un caso real de relevancia. Se trata del mercado municipal de Algeciras que es una de las construcciones más importantes de EduardoTorroja, mundialmente conocida, que está siendo considerada para su declaración como monumento histórico-artístico y que ha sido objeto de una reciente Investigación con la finalidad de conocer el estado actual de la estructura y determinar las posibles causas que han originado el deterioro de la misma (Foto 2). En consecuencia, en los objetivos anteriores se prestará especial atención a los materiales y técnicas de reparación que se prevé usar en el demostrador.

Los resultados del proyecto permitirán mejorar el conocimiento de las características de estos materiales y proponer formulaciones más adecuadas a los requerimientos propios

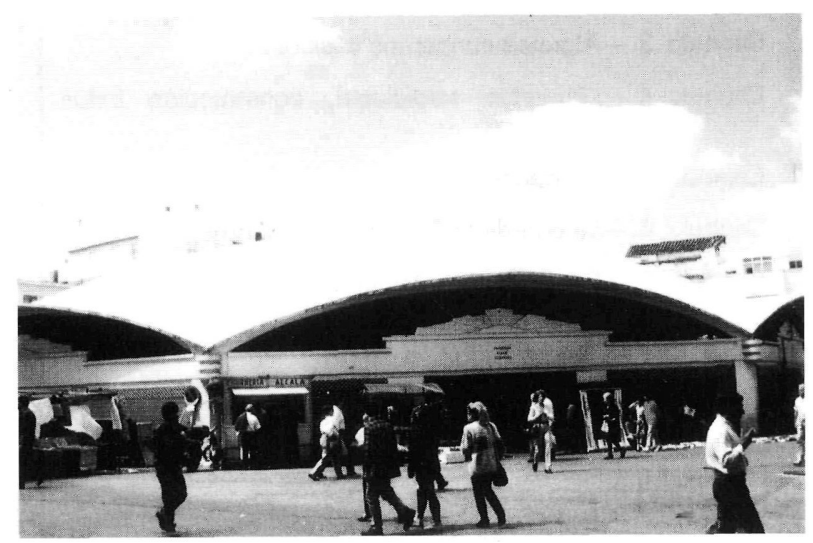

Foto 2.- Mercado de Algeciras. de la construcción, de los adhesivos apropiados, de los mecanismos de transferencia de cargas de los elementos estructurales antiguos a los nuevos, su evolución con el tiempo y las condiciones ambientales, poner a punto normas para proyectar y la tecnología para aplicar el refuerzo, durabilidad, implicaciones económicas y de puesta en obra. Con ello se espera abrir la Industria de la Construcción a los materiales compuestos de uso estructural ya utilizados en otras industrias (aeronáutica, naval, automovilística, ferroviaria, etc.), estimulando el desarrollo innovador e industrializando las tecnologías de refuerzo, mejorando su fiabilidad y durabilidad y abaratando sus costes totales.

\section{BIBLIOGRAFÍA COMENTADA}

(1) W. TSAI, STEPHEN,: "Theory of Composites Design", Ed. Think Composites, 1992, USA. Se presentan las bases para el análisis teórico de estructuras de materiales compuestos.

(2) MIRAVETE, A.: "Los nuevos materiales en la construcción”, Ed. A. Miravete, 1994, Zaragoza. Se presenta una amplia panorámica de las posibilidades que ofrecen los materiales compuestos en la construcción, con gran número de ejemplos y una descripción de las características de estos materiales.

(3) Composites in infrastructure design and rehabilitation, NAFEMS Research Working Group Seminar, Ed. V Peshkam, Mouchel Engineering, Science \& Technology, November 1995, London U.K. Presenta una serie de consideraciones teóricas, de realizaciones y de estudios sobre refuerzo de estructuras con materiales compuestos, llevados a cabo en distintos países de todo el mundo.

(4) MEIER, URS: "Advanced composites for structural repair: European perspective", Advanced Materials and Techniques, pág. 491-503, Barcelona 96. Presenta un análisis de la utilización de los materiales compuestos en la reparación y rehabilitación de estructuras en Europa. Propone indicaciones sobre su uso e indica la cifra de 10.000 millones de dólares USA como ahorro que podría producirse en Europa con el uso de estos materiales en el refuerzo de estructuras, aproximadamente la mitad sólo en puentes.

(5) Report on Japan-Canada Workshop on Advanced Composites Materials for Concrete Structures, marzo 1996. Presenta las conclusiones de la última reunión entre técnicos de Japón y de Canadá para discutir sobre la elaboración de códigos de diseño de estructuras de materiales compuestos.

(6) MALVAR, L.J., WARREN G.E. and INABA C.M.: "Composites applications in the Navy Waterfront Infrastucture". Materials for the New Millenium, 4th ASCE Materials Conference, noviembre 1996, Whashington. Presenta los resultados de la utilización experimental de materiales compuestos en el refuerzo de instalaciones marítimas, realizados en los laboratorios de la Navy, en San Diego, EE.UU 
(7) C. BANK LAURENCE et al.,; International Research on Advanced Composites for Construction, IRACC 96. US Research Proyect. Presenta una amplia relación de los distintos proyectos de investigación sobre el uso de materiales compuestos en construcción, llevadas a cabo en distintas universidades de EE.UU.

(8) Composite structures: conventional and innovative. IABSE symposium, Inssbruck, 1997. Presenta aportaciones con- ceptuales, casos de aplicación y noticia de proyectos en desarrollo en todo el mundo.

(9) Materiales Compuestos en la Construcción: Materiales de Construcción, $\mathbf{n}^{\text {os }} 247-248$, 1997. En este número de la revista se describen con detalle estos materiales, incidiendo en sus amplias e importantes aplicaciones en la Construcción.

\section{Publicaciones del Instituto Eduardo Torroja-CSIC}

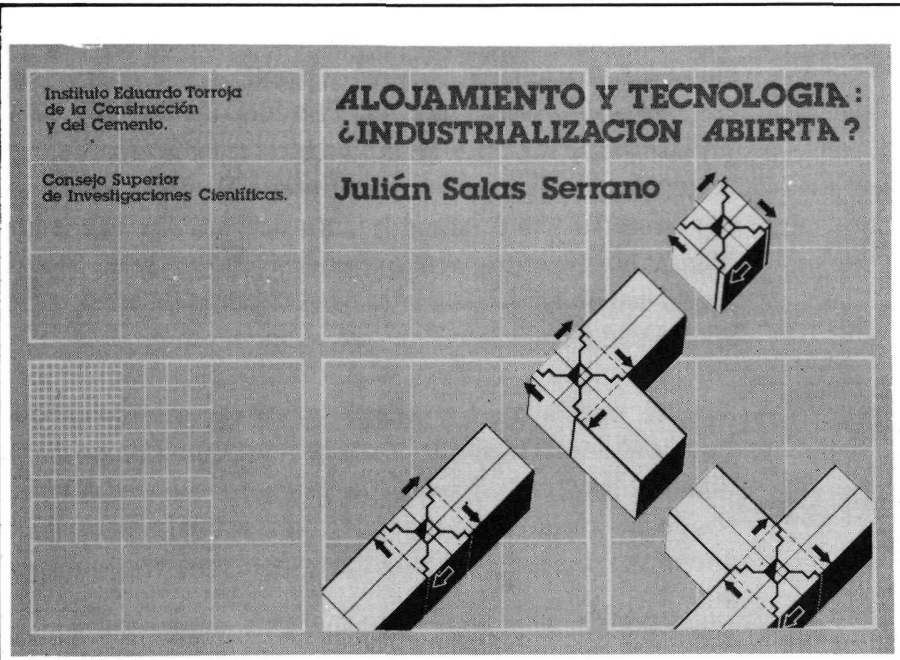

ALOJAMIENTO Y TECNOLOGIA: ¿INDUSTRIALIZACION ABIERTA?

JULIAN SALAS, ING. IND. (I.E.T.c.c.)

Un volumen de 160 páginas, 109 figuras y 16 tablas. Tamaño $240 \times 168 \mathrm{~mm}$. Encuader̂nado en rústica.

\section{SUMARIO:}

Prólogo Prof. G. Ciribini.

\section{Introducción}

Capítulo 1. - La industrialización en las proclamas y manifiestos de arquitectura.

Capítulo 2.-¿Réquiem por la construcción industrializada?

Capítulo 3. - Algunos conceptos básicos.

Capítulo 4.-¿Proyecto tradicional, construcción industrializada?

Capítulo 5.-Componentes.

Capítulo 6. - La coordinación dimensional hoy.

Capítulo 7.-Flexibilidad, intercambiabilidad y catálogos.

Capitulo 8.-Industrialización, normativa y calidad.

Capítulo 9.-Reflexiones finales.

publicación del

INSTITUTO EDUARDO TORROJA 